\title{
D-Riboce-L-Cystein Maintained Testicular Integrity in Rats Model (Rattus Novergicus) Exposed to X-Ray
}

\author{
Dare Babatunde Joseph ${ }^{1,}$, , Olayemi Olamide Samuel ${ }^{1}$, Falana Benedict Abiola ${ }^{1}$, \\ Duri Francis I. Ogueri ${ }^{2}$, Osinubi Abraham A. A. ${ }^{2}$ \\ ${ }^{1}$ Anatomy Department, Osun State University, Osogbo, Nigeria \\ ${ }^{2}$ Anatomy Department, University of Lagos, Lagos, Nigeria
}

Email address:

babatunde.dare@uniosun.edu.ng (D. B. Joseph)

${ }^{*}$ Corresponding author

\section{To cite this article:}

Dare Babatunde Joseph, Olayemi Olamide Samuel, Falana Benedict Abiola, Duri Francis I. Ogueri, Osinubi Abraham A. A. D-Riboce-LCystein Maintained Testicular Integrity in Rats Model (Rattus Novergicus) Exposed to X-Ray. Cell Biology. Vol. 5, No. 4, 2017 , pp. 38-44. doi: $10.11648 /$ j.cb.20170504.12

Received: March 12, 2017; Accepted: May 27, 2017; Published: July 18, 2017

\begin{abstract}
Oxidative stress mechanisms are involved in xenobiotic-induced testicular dysfunctions which consequently lead to male infertility, however, antioxidants work like a defense system, disarming free radicals. Therefore, this study is aimed at investigating the maintenance of testicular integrity using D-Riboce-L-cysteine on X-ray induced testicular damage in adult wistar rats (Rattus Novergicus). A total of 20 male rats were randomly selected into Five (5) groups of Four (4) animal each. Control animals received only water while treated animals include: animals induced with X-ray only; around the pelvic and perineum region at about $95 \mathrm{kv}, 12.5$ milliampere-seconds (mA.s), 50 focal field distance (FFD), animals treated with DRiboce-L-cysteine at $30 \mathrm{mg} / \mathrm{kg}$ body weight of D-Riboce-L-cysteine before exposed to $95 \mathrm{kv}, 12.5$ milliampere-seconds (mA.s), 50 focal field distance (FFD) of X-ray, animals treated with 95kv, 12.5 milliampere-seconds (mA.s), 50 focal field distance (FFD) of X-ray per animal before receiving $30 \mathrm{mg} / \mathrm{kg}$ body weight of D-Riboce-L-cysteine and animals treated with $30 \mathrm{mg} / \mathrm{kg}$ body weight of D-Riboce-L-cysteine only. After 21 days of treatments, the animals were sacrificed, and the testes were excised following abdominal incision, fixed in Bouin's fluids for histological observations and right testis was homogenized in 5\% sucrose solution for determination of enzymes of carbohydrates metabolism. Sperm was obtained from the caudal part of the epididymis for analysis of sperm characteristics. Reduced sperm count, abnormal morphology and significant $(\mathrm{p}<0.05)$ higher non motile sperm characterized the animals expose to X-ray. However, sperm characteristics was maintained in control animals $(\mathrm{p}<0.05)$ and animals treated with D-Riboce-L-cysteine only. Reduced activities in enzyme of carbohydrate metabolism (G-6-PDH) and significant increase in the level of lipid peroxidation shown by the activities of MDA in the X-ray treated groups compared to animals treated with D-Riboce-L-cysteine $(p<0.05)$ and the control animals. Abnormal widening of the interstitial space, loss of the basal laminal, degeneration in spermatogonia with vacuolation. Loss of germinal epithelium of the seminiferous tubules were also observed in animals expose to X-ray. Exposure to X-ray disrupts spermatogenesis by disruption and depletion of the spermatids and spermatogonia population, which caused increase in testicular tissue damage and consequently, altered the sperm characteristics. D-Riboce-L-cysteine clearly demonstrated maintenance of testicular integrity and enhance sperm characteristics; indication of fertility enhancing ability.
\end{abstract}

Keywords: X-Ray, Testes, Free Radicals, Antioxidants and Wistar Rats

\section{Introduction}

With the development of nuclear technique, human beings are facing more dangerous effects of using ionizing radiation in different aspects of modern life than before [1]. Ionizing radiation inflicts its adverse effects through the generation of oxidative stress that unleash large-scale destruction or damage of various biomolecules. Oxidative stress mechanisms are 
involved in xenobiotic-induced testicular dysfunctions which consequently lead to male infertility [2]. Under normal conditions, the testis is afforded with antioxidants protection as an elaborate array of antioxidants enzymes, free radical scavengers, and low oxygen tension in order to support the wellbeing of the testes and leydig cells steroidogenic function [2]. However, a wide variety of endogenous and exogenous factors are known to compromise male fertility by generating free radicals in testes [3]. In order to prevent and relieve the hazard to human reproductive health induced by ionizing radiation exposure, avoid the toxicity and the side effects and for the labialization of anti-radiation drugs [4]; Radioprotectors derived from traditional foods and medicinal plant sources, is worthy to receive great attention and special consideration. Free radicals are atoms or groups of atoms with an odd (unpaired) number of electrons and can be formed when oxygen interacts with certain molecules, which once formed these highly reactive radicals initiate chain reaction. Radiation react with important cellular components such as DNA, or the cell membrane and alter cells functions leading to cell death. Therefore, to prevent free radical damage, the body has a defense system of antioxidants. It is known that free radicals play a fundamental role in several diseases [5]. Ionizing radiation carries more than $10 \mathrm{eV}$, which is enough to ionize atoms and molecules, and break chemical bonds. X-rays are electromagnetic waves with a wavelength less than about $10^{-9} \mathrm{~m}$ (greater than $31017 \mathrm{~Hz}$ and 1,240 eV). Reactive oxygen species (ROS) are chemically reactive chemical species containing oxygen. However, during times of environmental stress (UV or heat exposure), ROS levels can increase dramatically result in significant damage to cell structures [6] known as oxidative stress. Oxidative stress is associated with increased production of oxidizing species or a significant decrease in the effectiveness of antioxidants defense, such as glutathione [7]. The testes have several protective mechanisms that minimize the toxic potential of these reactive oxygen species to ensure that the twin processes spermatogenic and steroidogenic functions of this organ are not impacted by oxidative stress [8].

D-Riboce-L-cysteine is one of the most important components to having healthy fertility that every woman and man needs to focus on. Antioxidants are a family of vitamins, minerals and other nutrients that protect the body from the damage caused by free radicals [8]. Antioxidants work like a defense system, disarming free radicals. They are a kind of police force within the body. Antioxidants "quench" free radicals and prevent the spread of ROS that cause damage to cells [9]. Radiations have been shown to exert carcinogenic effect in humans and experimental animals [10]. Generation of reactive oxygen species (ROS) and its interference with cellular antioxidants system is one of the major mechanisms by which carcinogenic effect is mediated [11]. As a result of its high natural vitamin $\mathrm{C}$ content, D-Riboce-L-cysteine has a well remarkable antioxidants capability [12]. Radiation acts as a catalyst in forming reactive oxygen species. It increases lipid peroxidation; in addition it depletes glutathione and proteinbound sulfhydryl groups. It also promotes the production of inflammatory cytokines [13]. Riboceine is a unique molecule that combines ribose and cysteine, nutrients that occur naturally in the body [14]. Riboceine once ingested will be absorbed, enters the bloodstream and delivers cysteine and ribose to the cells, supporting glutathione production as well as providing ribose, an integral part of ATP, cells natural fuel and source of energy. Riboceine significantly out formed other means of glutathione enhancement [14]. This research project is aimed to study the effect of D-Riboce-L-cysteine on radiation (X-ray)-induced oxidative stress in rat testes.

\section{Materials and Methods}

\subsection{Animal Source and Handling}

Twenty [20] adult male wistar albino rats each weighing between $50-80 \mathrm{~g}$ were procured from Jumorak Vetinary Center (JVC) Iwo, Osun State. The rats were kept in the animal control room of Osun State University and acclimatized for 2 weeks till they attained weight of $100-$ $150 \mathrm{~g}$. The rats were fed on starter mash (Vital Feeds Grand Cereals Ltd. Ibadan); water was given ad libitum and maintained under standard conditions. The animal room was well ventilated with a temperature range of 25-27 under day/night 12-12hour photoperiodicity. The rats were randomly selected into five (5) groups of four (4) rats each; Group One (Control), Two (radiation only), Three (antioxidants before radiation), Four (radiation before antioxidants) and Five (antioxidants only).

\subsection{Drug Preparation}

D-Riboce-L-cysteine was obtain from Lagos State Teaching Hospital, Lagos State. Ten capsule of D-Riboce-Lcysteine $(1250 \mathrm{mg})$ was dissolved in $125 \mathrm{ml}$ of distilled water for 24 hours. It was refrigerated throughout the experimental period of 21 days.

\subsection{Administration}

Administration of D-Riboce-L-cysteine was done by oral using oral cannula. The animals (Group two and four) were exposed once to X-ray around the pelvic and perineum region at about $95 \mathrm{kv}, 12.5$ milliampere-seconds (mA.s), 50 focal field distance (FFD) per animal. The animals (group three and five) received $30 \mathrm{mg} / \mathrm{kg}$ D-Riboce-L-cysteine per body weight each in the first day. Administration of $30 \mathrm{mg} / \mathrm{kg}$ D-Riboce-L-cysteine per body weight were done daily for 21days at 9:00a.m.

Group A (control group) received water and starter mash only, Group B (radiation only) received 95kv, 12.5 milliampere-seconds (mA.s), 50 focal field distance (FFD) of $\mathrm{X}$-ray per animal. Group $\mathrm{C}$ received $30 \mathrm{mg} / \mathrm{kg}$ body weight of D-Riboce-L-cysteine before receiving $95 \mathrm{kv}$, 12.5 milliampere-seconds (mA.s), 50 focal field distance (FFD) of $\mathrm{X}$-ray per animal. Group D received $95 \mathrm{kv}, 12.5$ milliampereseconds (mA.s), 50 focal field distance (FFD) of X-ray per animal before receiving $30 \mathrm{mg} / \mathrm{kg}$ body weight of D-RiboceL-cysteine, Group E received $30 \mathrm{mg} / \mathrm{kg}$ body weight of D- 
Riboce-L-cysteine only.

\subsection{Animal Sacrifice}

The animals were sacrifice 24 hours after last administration. Blood was taken from the heart. Animals were sacrificed by ethyl ether and the testes were excised following abdominal incision and fixed in $10 \%$ formal-saline for histology analysis. The epididymis was fixed in normal saline for sperm count, motility and morphology. The testes were homogenized in $5 \%$ sucrose solution for enzymes assay.

\subsection{Routine Histological Preparation}

\subsubsection{Sperm Characteristics}

\section{a). Sperm count}

The concentration of spermatozoa was determined using the haemocytometer method [15]. In this procedure a 1:20 dilution from each well-mixed sample was prepared by diluting $50 \mu 1$ of liquefied semen with $950 \mu 1$ diluents. The diluents was prepared by adding $50 \mathrm{~g}$ of sodium carbonate $\left(\mathrm{NaHCO}_{3}\right), 10 \mathrm{ml}$ of $35 \%(\mathrm{v} / \mathrm{v})$ formalin and $0.25 \mathrm{~g}$ of trypan blue to distilled water and making up the solution to a final volume of $1000 \mathrm{ml}$. A fixed volume of the sample was withdrawn with micro-pipette and delivered onto the edges of Neubauer chamber of the heamocytometer and covered with a $2222 \mathrm{~mm}$ cover slip. The weight of the cover slip spread the sample which made the semen to move to the center of the Neubauer center by capillary action and standardized so that the analyses were carried out in a preparation with fixed depth.

Both chambers of the heamatocytometer were scored and the average count was calculated, provided that the difference between the two counts did not exceed 1/20 of their sum (less than $10 \%$ difference). If the two counts were not within $10 \%$, they were discarded, the sample dilution re-mixed and another haemocytometer prepared and counted.

b) Analysis of the Sperm morphological characteristics

Sperm cell represent a unique population in which up to $50 \%$ (up to $70 \%$ according to WHO criteria 1992 and up to $86 \%$ according to strict criteria) of the cells can have morphological defects in normal fertile individuals. The normal head should be oval in shape.

Thus the sperm morphological characteristics were graded based on the following parameters;

a. Slight shrinkage that fixation induce, the length of the head about 4.0-5.5 $\mu \mathrm{m}$, and the width $2.5-3.510 \mu \mathrm{m}$. the length-to-width ratio about 1.50 to 1.75 .

b. There should be a well-defined acrosomal region comprising $40-70 \%$ of the head area.

c. There must be no neck, mid-piece or tail defects and no cytoplasm droplet more than one-third the size of a normal sperm head. These classification schemes require that all borderline forms be considered abnormal [15].

The following categories of defects were scored.

a. Head shape/size defects, including large, small, tapering, pyriform, amorphous, vacuolated $(>20 \%$ of the head are occupied by unstained vacuolar areas), or double heads, or any combination of these.

b. Neck and midpiece defects, including absent tail, non inserted or bent tail (the tail forms an angle of about to the long axis of the head), distended/irregular/bent midpiece, abnormally thin midpiece or any combination of these.

c. Tail defects, including short, multiple, hairpin, broken, irregular width, or coiled tails, tails with terminal droplets, or any combination of these.

d. Cytoplasmic droplets greater than one-than area of a normal sperm head.

c). Sperm Motility

A fixed volume of semen was collect from harvested epididymis and put in normal saline. Not more than $10 \mu 1$ of the semen was withdrawn with micro-pipette and delivered onto a clean glass slide covered with a $2222 \mathrm{~mm}$ cover slip and standardized so that the analyses were carried out in a preparation with fixed depth (i.e., $20 \mu 1)$. This depth allowed full expression of the rotating movement of normal spermatozoa (WHO, 1992). The weight of the cover slip spread the sample for optimal viewing. The freshly made, wet preparation was left to stabilize for approximately one minute and the procedure was carried out at a room temperature between 18 and in the laboratory.

The microscopic field was scanned systematically and the motility of each spermatozoon was graded has been motile or non-motile.

Spermatozoa graded motile were supposed to display rapid progressive motility along a linear track, covering a distance of at least half the length of a spermatozoon per second.

a. Visual field close to the border of the cover slip should be avoided [15].

b. The animals blood were collected from the right ventricle and immediately put inside heparinized bottles for Serum-testosterone level analysis.

\subsubsection{Enzyme Histo-Chemistry}

Excised testicular tissues were put in homogenizer with $1 \mathrm{ml}$ of $5 \%$ sucrose solution and homogenized properly. Tissue homogenates were collected in $5 \mathrm{ml}$ plain serum bottle for enzyme assay; Glucose-6-phosphate dehydrogenase (G6PD), Malondialdehyde (MDA) and Glutathione Peroxidase levels.

\section{Results}

Table 1: Shown standard error of mean and P-value results for Sperm analysis (Microscopic sperm count $\{$ cell $/ \mathrm{ml}\}$, sperm morphology $\{$ Normal/Abnormal \%\} and sperm motility \{Motile/Non-motile \% $\}$ ).

Sperm count from table 1: showed that Sperm characteristics were significantly altered in the animals exposed to X-ray radiation; sperm counts was significantly reduced in the animals that was exposed only to X-ray radiation, morphology observation showed significant percentage increase in the abnormal sperm morphology and 
reduced or altered motility. However, control animals showed higher sperm counts relative to the animals that were exposed. Significant increase in sperm counts was observed in the animals that were treated with antioxidant extracts.

Animals exposed to both radiation and the antioxidants extracts showed marked improvement in the sperm characteristics that were observed over the animals exposed without administration of antioxidant. Increased sperm counts, morphological and motility integrity was maintained in the control animals, but significant increased in sperm counts, motility and morphology was noticed as shown in table 1 below

Table 1. Semen analysis.

\begin{tabular}{|c|c|c|c|c|c|}
\hline Parameter & $\begin{array}{l}\text { Group One } \\
\text { (Control) } \\
\text { Mean } \pm \text { SEM } \\
\end{array}$ & $\begin{array}{l}\text { Group Two } \\
\text { (Radiation Only } \\
\text { Mean } \pm \text { SEM } \\
\end{array}$ & $\begin{array}{l}\text { Group Three } \\
\text { (Antioxidant }+ \text { Radiation) } \\
\text { Mean } \pm \text { SEM }\end{array}$ & $\begin{array}{l}\text { Group Four (Radiation+ } \\
\text { Antioxidant) Mean } \pm \\
\text { SEM }\end{array}$ & $\begin{array}{l}\text { Group Five } \\
\text { (Antioxidant Only) } \\
\text { Mean } \pm \text { SEM }\end{array}$ \\
\hline \multirow{2}{*}{ Sperm Count $\left(10^{6}\right.$ cell $\left./ \mathrm{ml}\right)$} & \multirow{2}{*}{$690.0 \pm 10.00$} & $320.0 \pm 20.00$ & $470.0 \pm 10.00$ & $500.0 \pm 20.00$ & $710.0 \pm 10.00$ \\
\hline & & $* * 0.0036$ & $* * 0.0041$ & $* 0.0136$ & $* * 0.0033$ \\
\hline \multirow{2}{*}{ Normal Morphology (\%) } & \multirow{2}{*}{$87.50 \pm 2.500$} & $47.50 \pm 2.500$ & $62.50 \pm 2.500$ & $72.50 \pm 2.500$ & $89.00 \pm 1.000$ \\
\hline & & $* * 0.0077$ & $* 0.0194$ & s 0.0194 & $* * 0.0042$ \\
\hline \multirow{2}{*}{ Abnormal Morphology (\%) } & \multirow{2}{*}{$12.50 \pm 2.500$} & $52.50 \pm 2.500$ & $37.50 \pm 2.500$ & $27.50 \pm 2.500$ & $11.00 \pm 1.000$ \\
\hline & & $* * 0.0077$ & $* 0.0194$ & s 0.0194 & $* * 0.0042$ \\
\hline \multirow{2}{*}{ Sperm Motility (\%) } & \multirow{2}{*}{$82.50 \pm 2.500$} & $51.50 \pm 6.500$ & $37.50 \pm 2.500$ & $47.50 \pm 2.500$ & $86.00 \pm 1.000$ \\
\hline & & $* 0.0111$ & $* * 0.0061$ & $* 0.0101$ & $* * 0.0082$ \\
\hline \multirow{2}{*}{ Non- motile (\%) } & \multirow{2}{*}{$17.50 \pm 2.500$} & $70.00 \pm 5.000$ & $62.50 \pm 2.500$ & $52.50 \pm 2.500$ & $14.00 \pm 1.000$ \\
\hline & & $* 0.0111$ & 0.0061 & $* 0.0101$ & $* * 0.0082$ \\
\hline
\end{tabular}

*Significantly different at $\mathrm{p}<0.05$

Table 2. Serum level of enzymes of carbon hydrates metabolism (G-6-PDH), lipid peroxidation (MDA) and antioxidant enzymes (GPx) activities.

\begin{tabular}{|c|c|c|c|c|c|}
\hline & $\begin{array}{l}\text { Group One } \\
\text { (Control) Mean } \pm \\
\text { SEM }\end{array}$ & $\begin{array}{l}\text { Group Two } \\
\text { (Radiation Only } \\
\text { Mean } \pm \text { SEM } \\
\end{array}$ & $\begin{array}{l}\text { Group Three } \\
\text { (Antioxidant }+ \\
\text { Radiation) Mean } \pm \text { SEM } \\
\end{array}$ & $\begin{array}{l}\text { Group Four (Radiation }+ \\
\text { Antioxidant) Mean } \pm \\
\text { SEM }\end{array}$ & $\begin{array}{l}\text { Group Five (Antioxidant } \\
\text { Only) Mean } \pm \text { SEM }\end{array}$ \\
\hline \multirow{2}{*}{ G6PDH (IU/L) } & \multirow{2}{*}{$3916 \pm 26.00$} & $2112 \pm 104.0$ & $2668 \pm 208.0$ & $3023 \pm 61.00$ & $4002 \pm 16.50$ \\
\hline & & $* * 0.0035$ & $* 0.0271$ & $* 0.0055^{\mathrm{s}}$ & $* * 0.0031$ \\
\hline \multirow{2}{*}{$\operatorname{MDA}(\mu \mathrm{mol} / \mathrm{L})$} & \multirow{2}{*}{$19.50 \pm 0.5000$} & $32.00 \pm 1.000$ & $29.50 \pm 0.5000$ & $28.00 \pm 1.000$ & $17.50 \pm 0.5000$ \\
\hline & & 0.0079 & $* * 0.0050$ & $* 0.0169$ & $* 0.0059$ \\
\hline GP (IU/L) & $3259 \pm 104.5$ & $2237 \pm 157.0 *$ & $2484 \pm 366.0$ & $2514 \pm 38.00 *$ & $3409 \pm 82.50$ \\
\hline
\end{tabular}

*Significantly different at $\mathrm{p}<0.05$

$\mathrm{SEM}=$ Standard error of mean

MDA $=$ Malondialdehyde

$\mathrm{GPx}=$ Glutathione peroxidase

The enzyme results obtained were analyzed at 0.05 level of significance. Changes in tissue levels of Glutathione peroxidase (GP) and the activity of the enzymes glucose -6phosphate dehydrogenase (G6PDH) are presented in the table 2. G6PDH activity was significantly higher in the control group compared to all other groups. The enzyme activity was also higher in control group compared to group two (radiation only), the difference was significant. The enzyme activity was also significantly lower in group two (radiation only) compared to group four (radiation + antioxidant) and group five (antioxidant only). MDA level was lower in the control group compared to all other groups except group five (antioxidant only) which was higher although it was insignificant; other differences were only significant to group two, three and four. MDA level was higher in group two (radiation only) compared to all other group, although insignificant, however, MDA level was significant lower in group five (antioxidant only). GP level was higher in the control group compared to all other groups except group five which was higher although insignificantly different.

The photomicrograph of the testis of the control animals in group one showed basic histology arrangement of the testis with the testicular lobules, seminiferous epithelium and its constituent cells. Moreso, the testis of the animals in group two (X-ray only) showed widening of the interstitial space, loss of the basal laminal, degeneration in spermatogonia with vacuolation observed. Losses of germinal epithelium of the seminiferous tubules were also observed in X-ray disrupts spermatogenesis by destroying the spermatids and spermatogonia.

The photomicrograph of the testis of the animals in group three (D-Riboce-L-cysteine+ X-ray) also showed abnormal widening of the interstitial spaces, reduced leydig cells and degeneration of the spermatogonial cells showing vaculation.

Animals in group four (X-ray+ D-Riboce-L-cysteine) also showed abnormal widening of the interstitial spaces, reduced leydig cell and degeneration of the spermatogonial cell.

The photomicrograph of the testis of the animals in group five (D-Riboce-L-cysteine) showed an intact testicular integrity maintained with interstitial space, spermatogonia at different stages well expressed; an indication of fertility enhancing ability of D-Riboce-L-cysteine. 


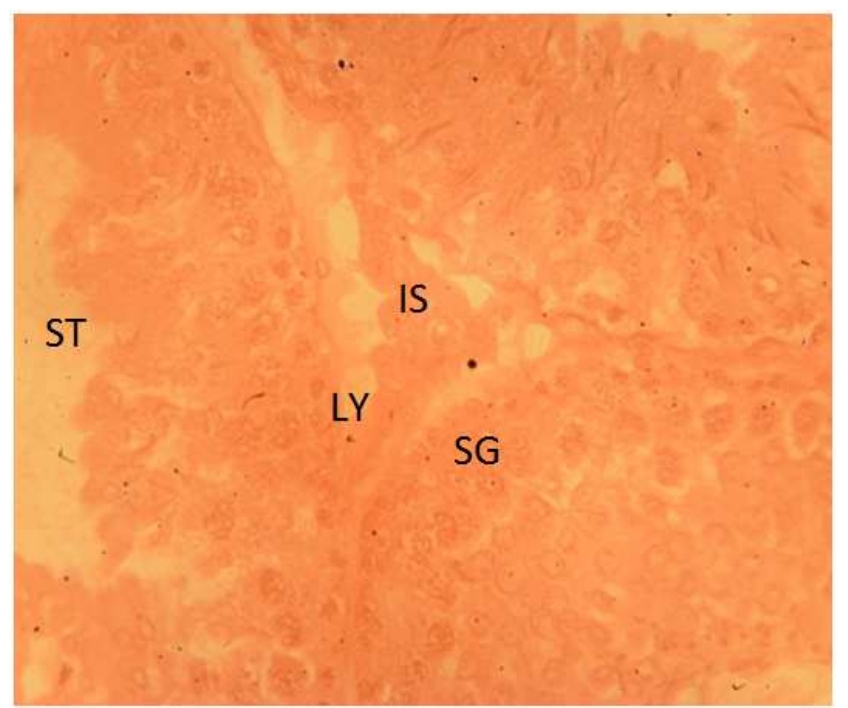

Figure 1. Testicular section of rats in control group stain with H/E X400; spermatogonia in the basal laminar, spermatogonia $A$ and $B$ are well expressed. The interstitial space was with intact leydig cell.

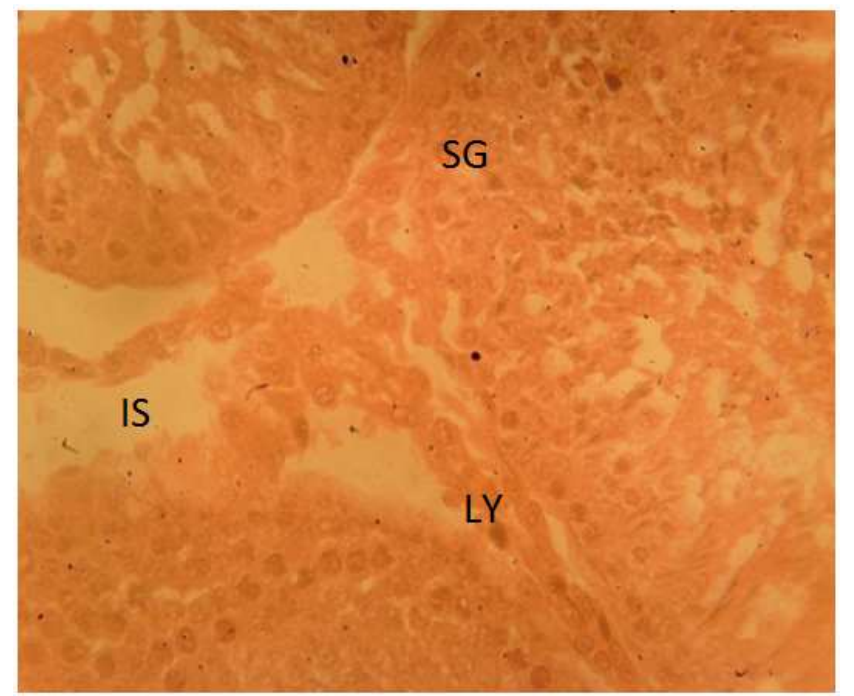

Figure 2. Testicular section of rats expose to radiation only, stain $H / E X$ 400, widening of the interstitial space, and loss of the basal laminal, degeneration in spermatogonia with vacuolation.

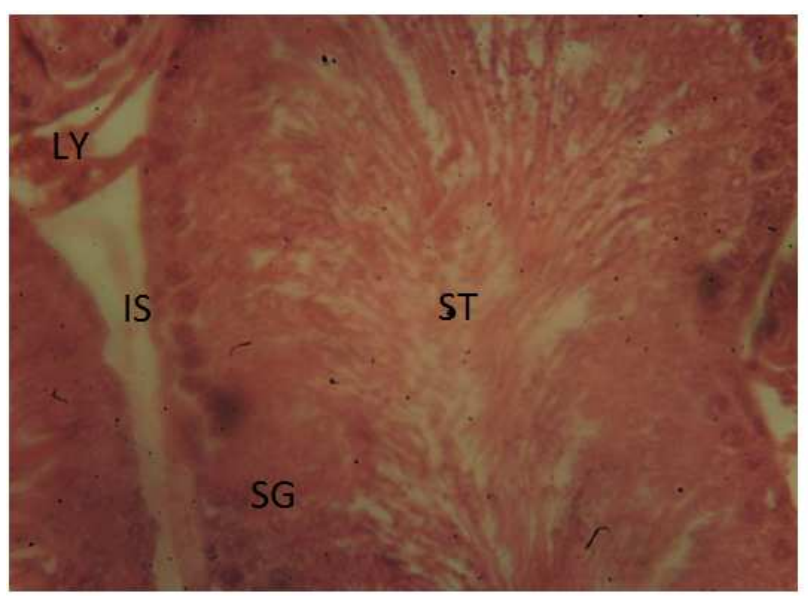

Figure 5. $A$ and B Testicular section of rats treated D-Riboce-L-cysteine stain with H/E X400 Intact testicular integrity was maintain with interstitial space, spermatogonia at different stages were well expressed.

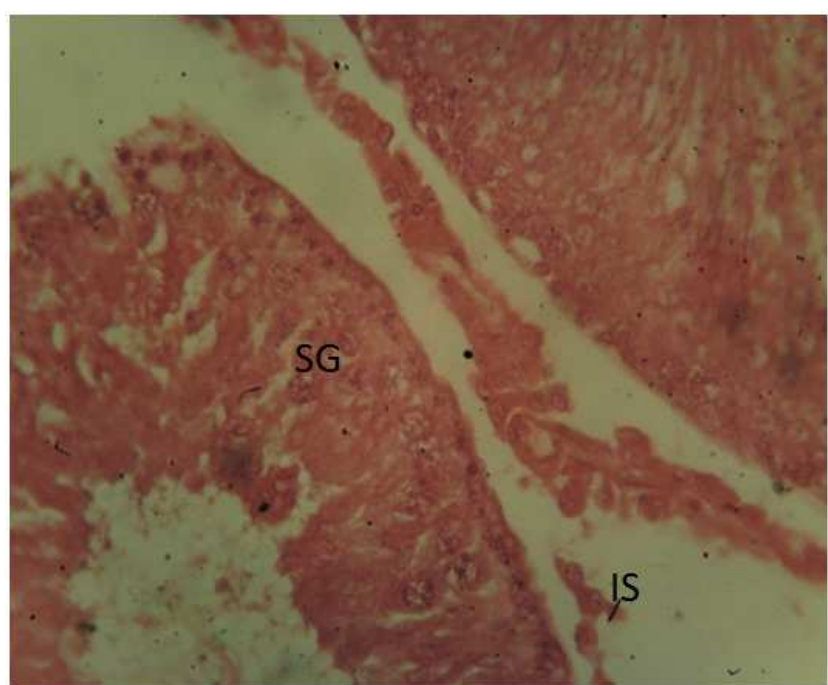

Figure 3. Testicular section of rats treated with D-Riboce-L-cysteine $+X$ ray stain with $H / E X 400$; widening of the interstitial spaces, intact leydig cells, spermatogonia population are well expressed with different stages revealed.

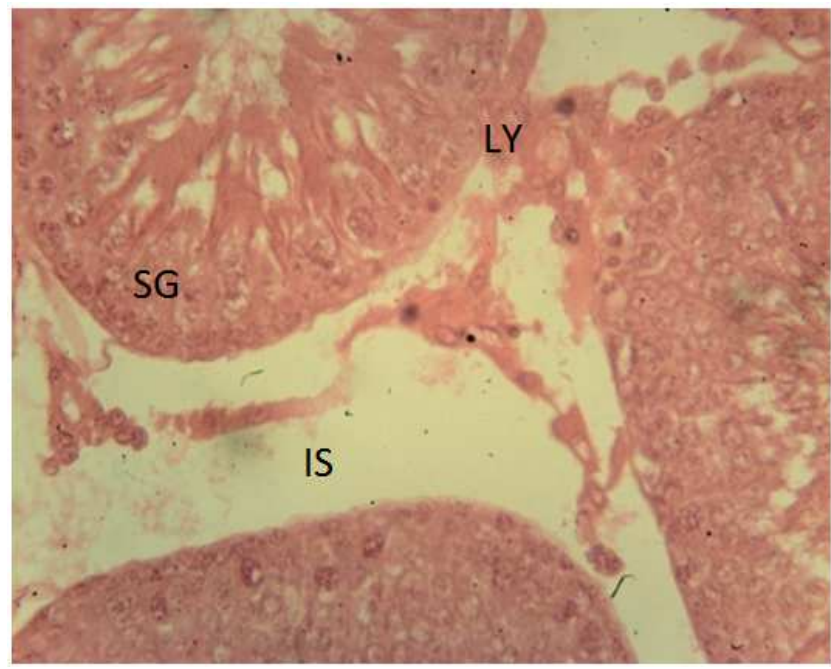

Figure 4. Testicular section of rats treated with X ray + D-Riboce-L-cysteine stain with $H / E X 400$; increased interstitial spaces, intact leydig cell and regeneration in spermatogonia population.

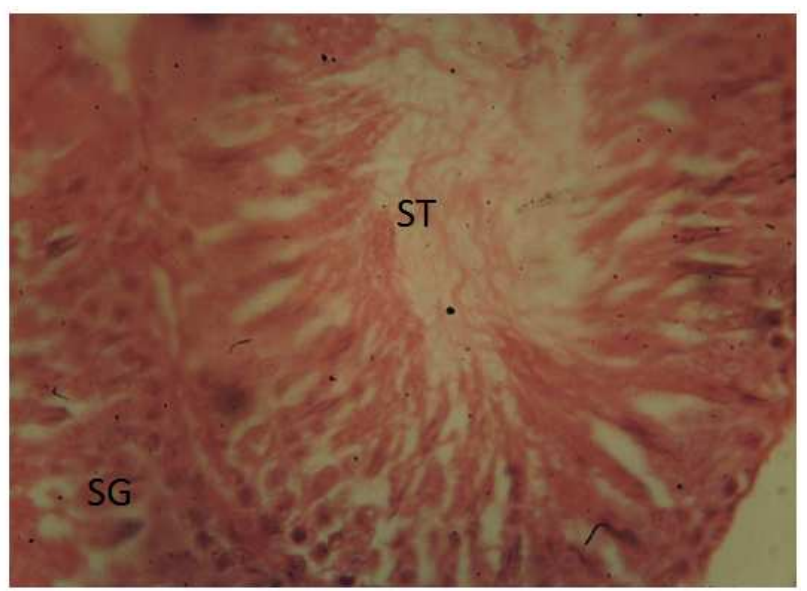




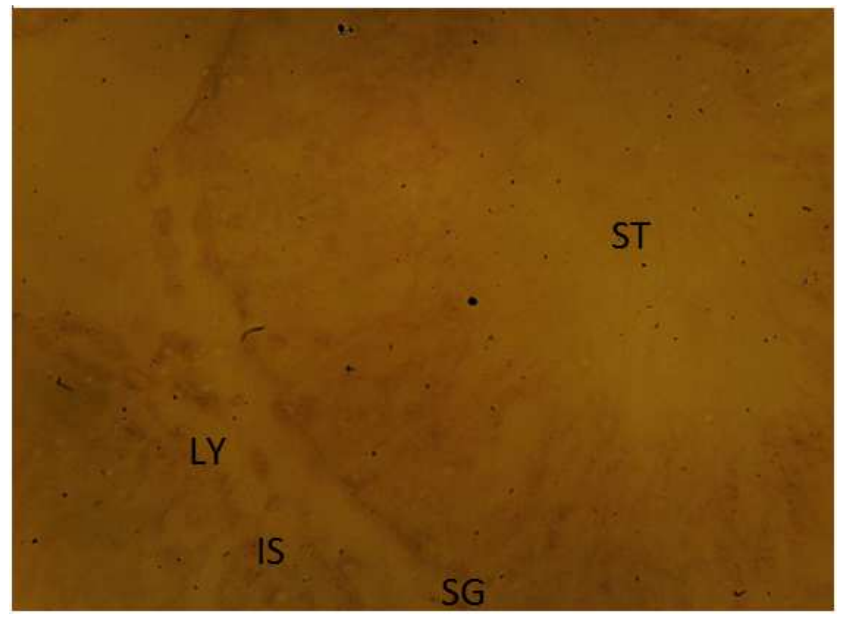

Figure 6. Testicular section of rats in control group stain with PAS X400, spermatogonia in the basal laminal, spermatogonia $A$ and $B$ are well expressed. The interstitial space was with intact leydig cell.

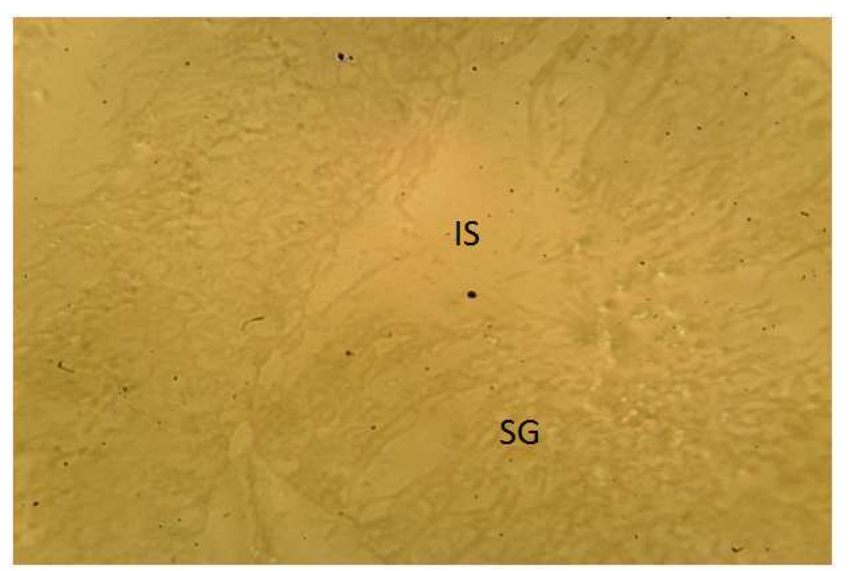

Figure 7. Testicular section of rats expose to radiation only, stain PAS X 400, widening of the interstitial space, loss of the basal laminal, reduced spermatogonia.

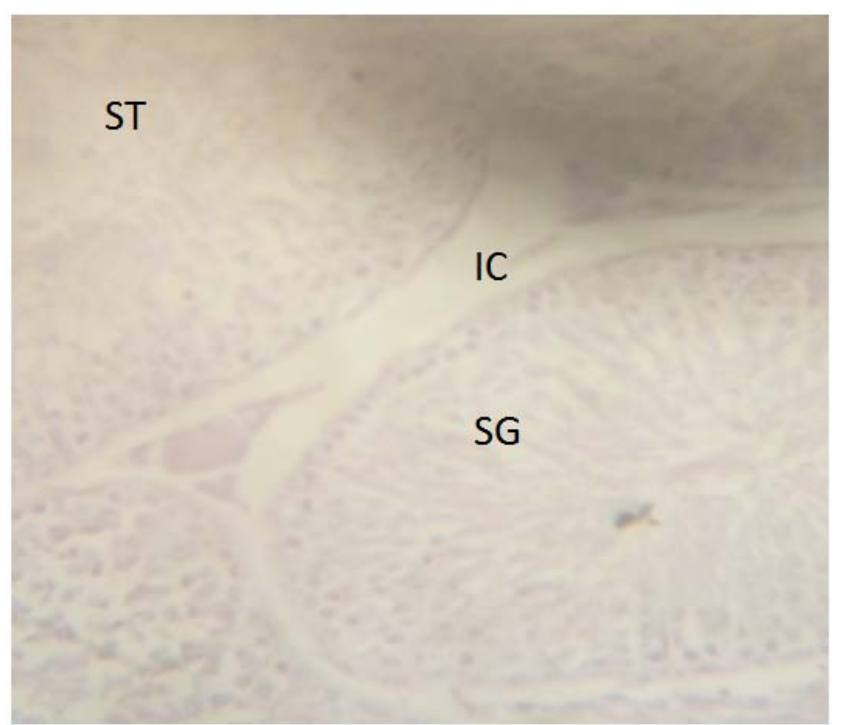

Figure 8. Testicular section of rats treated with D-Riboce-L-cysteine + X ray stain with PAS X400; reduced interstitial spaces, expression of spermatogonia from the basal membrane to the ad-luminal area showing all the stages.

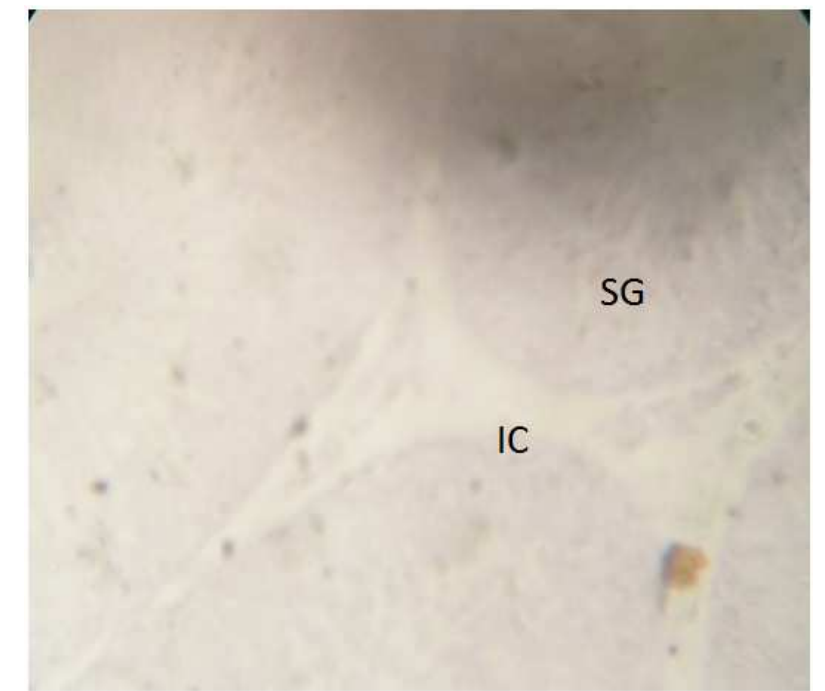

Figure 9. Testicular section of rats treated with D-Riboce-L-cysteine $+X$ ray stain with PAS X400 reduced interstitial spaces, intact leydig cell and regeneration of the spermatogonia.

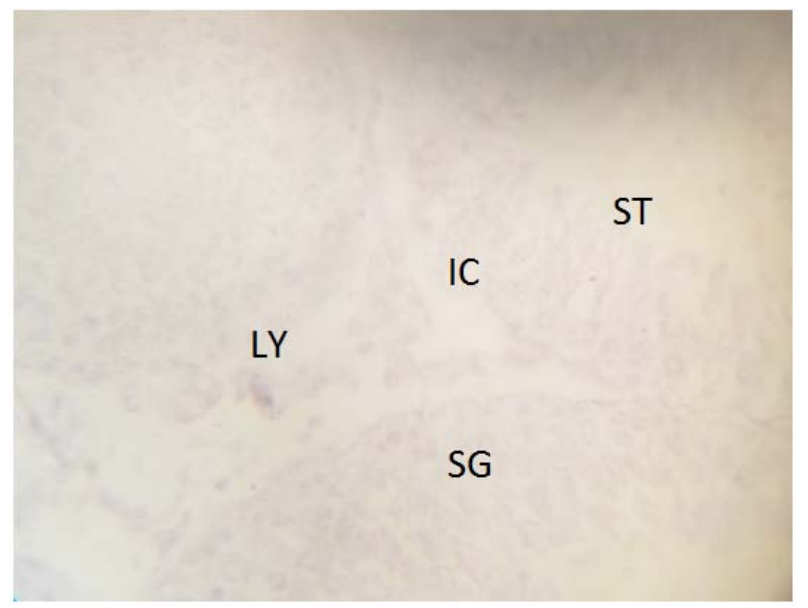

Figure 10. Testicular section of rats treated D-Riboce-L-cysteine stain with PAS X400 Intact testicular integrity was maintain with interstitial space, spermatogonia at different stages were well expressed.

\section{Discussion}

The development of radioprotective agents is important for protecting patients from the side-effects of radiotherapy, as well as occupational workers in nuclear and radiation plants. Natural compounds have been evaluated as radioprotectors and seem that they exert their effect through antioxidants content and immunostimulant activities.

In the present study, a decrease in testicular weight after radiation exposure was noticed. This decrease may be due to the actual loss in the germinal epithelial cells and not reflected by changes in the interstitial tissue or Sertoli cells.

The present study demonstrated that administration of DRiboce-L-cysteine on X-ray induced testicular damage of wistar rats caused moderation in the alteration in histological and histochemical parameters. Analysis of carbohydrate metabolic enzymes showed a highly significant decrease in activity of glucose-6-phosphate dehydrogenase (G-6-PDH) in 
X-ray only group as compared to control group; this reduction may be attributed to a compensatory response to oxidative stress where the consumption of G-6-PDH enzymes was to maintain sufficient levels of NADPH in response to the oxidative stress. However there was also a significant decrease in activity of glucose-6-phosphate dehydrogenase (G-6-PDH) in X-ray only group as compared to D-Riboce-L-cysteine only group and also significant decrease in activity of glucose-6phosphate dehydrogenase (G-6-PDH) was showed when compared to X-ray + D-Riboce-L-cysteine or D-Riboce-Lcysteine+ ray.

Estimation of end product of lipid peroxidation such as Malondialdehyde (MDA) is an index of oxidative damage to cellar structures [16]. Significant increase in MDA level in Xray group compared to control group showed are due to production of free radicals which causes damage and loss of functional properties. Tissue levels of Malondialdehyde (MDA) and glutathione peroxidase (GSH-P) are proven indicators of oxidative stress resulting from lipid peroxidation [17].

The decrease in sperm parameters in X-ray group compared to control group may be due to damage caused by oxidative stress in the tissue and also a significant increase in sperm parameters observed in D-Riboce-L-cysteine group may be as a result of antioxidant effect of D-Riboce-Lcysteine. It is believed that taking certain vitamins may help improve male fertility [18].

The exposure of rats to X-ray induces a biological and histological effects in the testes which can be attributed to increased oxidative stress resulting from radiation intoxication. D-Riboce-L-cysteine produces a regenerative effect against radiation damage and increase in G-6-PDH level as well as normal architecture of the testes; particularly in relation to the leydig cells and germinal epithelium, of the testes of the rat treated with D-Riboce-L-cysteine.

\section{Conclusion}

D-Riboce-L-cysteine has ability to play a protective role against radiation induced testicular damage. This study further ascertain fertility enhancing effects of D-Riboce-Lcysteine by maintaining the testicular functional and structural integrity.

\section{References}

[1] Alonzo, F., Hertel-Aas, T., Gilek, M., Gilbin, R., Oughton, D. H. and Garnier-Laplace, J. (2008): Modeling the propagation of effects of chronic exposure to ionizing radiation from individuals to populations. J. Environ. Radioact., 99: 14641473 .

[2] Doreswamy, K., Shrilatha, B., Rajeshkumar, T. and Muralidhara. (2004): Nickel-induced oxidative stress in testis of mice: evidence of DNA damage and genotoic effects. J. Androl., 25 (6): 996-1003.

[3] Hales, D. B., Allen, J. A. and Shankara, T. (2005): Mitochondrial function in Leydig cell steroidogenesis. Annals of the New York Academy of Sciences, 1061: 120-134.
[4] Ding, G. R. and Guo, G. Z. (2007): Advances in research of radioprotectant. J. Radiat. Res. Radiat. Process, 25: 321-324.

[5] Brigelius-Flohé R, Davies KJ (2007). "Is vitamin E an antioxidant, a regulator of signal transduction and gene expression, or a 'junk' food? Comments on the two accompanying papers: "Molecular mechanism of alphatocopherol action" by A. Azzi and "Vitamin E, antioxidant and nothing more" by M. Traber and J. Atkinson." Free Radical Biology \& Medicine 43 (1): 2-3.

[6] Dubrovsky BO (2005). Steroids, Neuroactive Steroids and Neurosteroids in Psychopathology. Prog.

Neuropsychopharmacol. Biol. Psychiatr. 29: 169-192.

[7] Gaballa A, Newton GL, Antelmann H, Parsonage D, Upton H, Rawat M, Claiborne A, Fahey RC, Helmann JD (2010). "Biosynthesis and functions of bacillithiol, a major lowmolecular-weight thiol in Bacilli." Proceedings of the National Academy of Sciences of the United States of America 107 (14): 6482-6.

[8] Peng F, Zhang B, Zhong, Li J, u G, Hu, Qiu W, Pei Z (2008). "Serum uric acid levels of patients with multiple sclerosis and other neurological diseases." Multiple Sclerosis 14 (2): 18896.

[9] Sharma, M. and Kumar, M. (2007): Radioprotection of Swiss albino mice by Myristica fragrans houtt. J. Radiat. Res., 48: 135-141.

[10] Ogbera, A. O., Dada, O., Adeyeye, F. and Jewo, P. I. (2010): Complementary and alternative medicine use in diabetes mellitus. West Afr. J. Med., 29 (3): 158-162.

[11] Aitken, R. J. and De Iuliis, G. N. (2007): Origins and consequences of DNA damage in male germ cells. Reproductive Bio-Medicine Online, 14 (6): 727-733.

[12] Obadoni BO, Ochuko PO (2001). Phytochemical Studies and Comparative Efficacy of the Crude Extracts of some Homeostatic Plants in Edo and Delta States of Nigeria, Glob. J. Pure Appl. Sci. 8: 203-208.

[13] Tan D, Manchester LC, Reiter RJ, Qi WB, Karbownik M, Calvo JR (2000). "Significance of melatonin in antioxidative defense system: reactions and products." Biological Signals and Receptors 9 (3-4): 137-59.

[14] Gehlot, P. and Goyal, P. K. (2007): Rectification of radiationinduced damage in swiss albino mice by aloe vera leaf extracts (AVE)," Iran. J. Rad. Res., 5 (2): 71-78.

[15] Packer L, Weber SU, Rimbach G (2001). "Molecular aspects of alpha-tocotrienol antioxidant action and cell signaling." The Journal of Nutrition 131 (2): 369S-73S.

[16] Watanabe S, Kang DH, Feng L, Nakagawa T, Kanellis J, Lan H, Mazzali M, Johnson RJ (2002). "Uric acid, hominoid evolution, and the pathogenesis of salt-sensitivity." Hypertension 40 (3): 355-60.

[17] Reiter RJ, Paredes SD, Manchester LC, Tan D (2009). "Reducing oxidative/nitrosative stress: a newly-discovered genre for melatonin." Critical Reviews in Biochemistry and Molecular Biology 44 (4): 175-200.

[18] Malhotra, N. and Devi, P. (2005): Radio protective Influence of Vitamin E on Energy Generating Enzymes in Prepubertal and Mature Rat Testis. Ind. J. Gerontology, 19 (1): 1-10. 